\title{
Influence du comportement élastique non-linéaire des conduites dans les écoulements transitoires avec cavitation de vapeur
}

\author{
LAMJED HADJ-TAÏEB ${ }^{\mathrm{a}}$ ET EzzEDDINE HADJ-TÄ̈EB \\ Mécanique des Fluides Appliquée et Modélisation, École Nationale d'Ingénieurs de Sfax, BP W 3038, Sfax, Tunisie
}

Reçu le 24 mai 2009, accepté le 10 mai 2010

\begin{abstract}
Résumé - Un modèle numérique permettant d'étudier l'influence du comportement élastique non-linéaire des conduites dans les écoulements transitoires avec cavitation de vapeur est présenté. L'application des lois de conservation de la masse et de la quantité de mouvement aboutit à un système de deux équations aux dérivées partielles de type hyperbolique qui se résout numériquement par un schéma prédicteur-correcteur aux différences finies conservatif. Par analogie avec la loi de Henry pour le dégazage de l'air dissous, une quantité de vapeur se dégage dès que la pression devient inférieure à la pression de vapeur du liquide. Pour mettre en évidence l'interaction fluide-structure, une relation liant la pression à la section de la conduite a été introduite. Les résultats obtenus permettent d'étudier l'influence de la déformation de la paroi de la conduite sur l'évolution des phénomènes de coup de bélier et de cavitation de vapeur. La formulation mathématique présentée est validée en comparant ces résultats à ceux expérimentaux tirés de la littérature.
\end{abstract}

Mots clés : Écoulement transitoire / cavitation de vapeur / conduite déformable / différences finies / interaction fluide-structure / loi non-linéaire

\begin{abstract}
Influence of non-linear elastic behaviour of pipes in transient flows with vapour cavitation. A numerical model is presented to study the influence of the non-linear elastic behaviour of the pipe wall in transient flows with vapour cavitation. The application of mass and momentum conservation laws yields to a system of two hyperbolic partial differential equations. The obtained system is resolved by a two-step finite differences numerical scheme. By analogy with the gas release phenomenon, when the liquid pressure in the pipe drops below the vapour pressure, vapour will be released from the liquid according to Henry's law. To put in evidence the fluid-structure interaction, a relationship between pressure and pipe section has been introduced. Computation results permit to study the influence of the pipe wall deformation on the evolution of water hammer and vapour cavitation phenomena. The presented mathematical formulation is validated while comparing these results to those experimental obtained from relevant literature.
\end{abstract}

Key words: Transient flow / vapour cavitation / flexible pipe / finite differences / fluide-structure interaction / non-linear law

\section{Introduction}

Les études classiques des écoulements transitoires en conduite sont menées généralement en considérant un comportement linéaire de la paroi de la conduite comme une hypothèse simplificatrice.

Cette hypothèse est acceptable dans le cas des conduites en métal et en béton, mais elle ne l'est plus dans le cas des conduites en plastiques (polyéthylène, PVC... ).

a Auteur pour correspondance : lamjed@quebecemail.com
Ces derniers ont un comportement différent de celui du métal et du béton.

Puisque l'utilisation des conduites en plastique est devenue de plus en plus fréquente dans plusieurs systèmes hydrauliques, ceci amène à examiner l'influence du comportement de ces matériaux sur les écoulements transitoires, essentiellement quand le phénomène de cavitation de vapeur apparaît.

Le problème de l'interaction fluide-structure et l'influence de la déformation de la paroi de la conduite sur l'évolution du phénomène de cavitation n'ont été que partiellement analysés par la prise en considération des 


\section{Nomenclature}

\begin{tabular}{|c|c|}
\hline$A$ & Section de la conduite $\left(\mathrm{m}^{2}\right)$ \\
\hline$B$ & Fonction \\
\hline$c$ & Facteur d'ancrage \\
\hline$C$ & Célérité $\left(\mathrm{m} . \mathrm{s}^{-1}\right)$ \\
\hline$D$ & Diamètre de la conduite (m) \\
\hline$E$ & Module de Young (Pa) \\
\hline$e$ & Épaisseur de la conduite (m) \\
\hline$F$ & Fonction \\
\hline$G$ & Fonction \\
\hline$H$ & Hauteur de pression $(\mathrm{m})$ \\
\hline$i$ & Indice de la discrétisation dans l'espace \\
\hline$k$ & Indice de la discrétisation dans le temps \\
\hline$L$ & Longueur de la conduite (m) \\
\hline$N$ & Nombre de tronçons \\
\hline$n$ & Exposant \\
\hline$N b$ & Nombre assez grand \\
\hline$p$ & Pression $(\mathrm{Pa})$ \\
\hline$R$ & Rayon de la conduite (m) \\
\hline$T$ & Température (K) \\
\hline$T_{\mathrm{m}}$ & Période mesurée de l'onde de pression \\
\hline$t$ & Temps (s) \\
\hline$t_{\mathrm{c}}$ & Temps de fermeture de la vanne (s) \\
\hline$U$ & Variable conservative \\
\hline$V$ & Vitesse $\left(\mathrm{m} . \mathrm{s}^{-1}\right)$ \\
\hline$W$ & Variable conservative \\
\hline$x$ & Distance $(\mathrm{m})$ \\
\hline$\alpha$ & Fraction volumique du liquide \\
\hline$\Delta x$ & Pas d'espace (m) \\
\hline$\Delta t$ & Pas de temps (s) \\
\hline$\delta$ & Paramètre de rigidité \\
\hline$\varepsilon_{\phi}$ & Déformation circonférentielle \\
\hline$\Gamma$ & Constante des gaz $\left(\mathrm{J} \cdot \mathrm{kg}^{-1} \cdot \mathrm{K}^{-1}\right)$ \\
\hline$\gamma$ & Inclinaison de la conduite \\
\hline$\lambda$ & Coefficient de frottement \\
\hline$\nu$ & Coefficient de Poisson \\
\hline$\rho$ & Masse volumique du fluide $\left(\mathrm{kg} \cdot \mathrm{m}^{-3}\right)$ \\
\hline$\sigma_{\phi}$ & Contrainte circonférentielle $(\mathrm{Pa})$ \\
\hline$\vartheta$ & Volume $\left(\mathrm{m}^{3}\right)$ \\
\hline & Indices \\
\hline 0 & Condition initiale \\
\hline 1 & Liquide \\
\hline $\mathrm{m}$ & Mélange \\
\hline $\mathrm{v}$ & Vapeur \\
\hline
\end{tabular}

propriétés géométriques et mécaniques des parois dans l'expression de la célérité supposée constante.

Nous développons dans cette étude un modèle de cavitation à bulles de vapeur dans les conduites élastiques à loi de comportement non-linéaire. L'objectif principal est d'effectuer une analyse par simulation numérique de l'influence de la déformation des parois de la conduite sur les écoulements transitoires avec cavitation de vapeur.

Pour étudier le phénomène de cavitation nous partons de l'idée générale qui dit que dès que la pression devient inférieure à la pression de vapeur du liquide il y a changement de phase et une quantité de vapeur se dégage selon une loi analogue à la loi de Henry pour le dégazage de l'air dissous [1]. En réalité, tout le liquide se transforme en vapeur. Une approche unidimensionnelle de l'écoulement du mélange liquide-vapeur est utilisée. La masse volumique du mélange est ainsi définie en fonction de la fraction volumique du liquide par une expression linéaire en la fraction volumique et non-linéaire en pression.

En considérant un comportement élastique nonlinéaire de la paroi de la conduite, les équations de conservation de la masse et de la quantité de mouvement du fluide sont réécrites sous une forme conservative. Le schéma aux différences finies de Lax-Wendroff est utilisé pour résoudre numériquement le système d'équations obtenu.

Les résultats obtenus permettent de tracer les courbes de pression et de vitesse en fonction du temps en différentes sections de la conduite. Le modèle proposé permet également d'étudier le comportement dynamique des conduites élastiques déformables et l'influence de l'élasticité sur l'évolution du phénomène de cavitation de vapeur. Ce modèle est validé en comparant les résultats de calcul à ceux de l'expérience.

\section{Formulation mathématique}

\section{1 Équations de mouvement}

Les équations de conservation de la masse et de la quantité de mouvement qui décrivent l'écoulement transitoire monodimensionnel dans les conduites peuvent être adoptées du modèle analytique développé par Streeter et Wylie [2] :

$$
\begin{gathered}
\frac{\partial(\rho A)}{\partial t}+\frac{\partial(\rho A V)}{\partial x}=0 \\
\frac{\partial V}{\partial t}+V \frac{\partial V}{\partial x}+\frac{1}{\rho} \frac{\partial p}{\partial x}=g \sin \gamma-\frac{\lambda V|V|}{2 D}
\end{gathered}
$$

où $\gamma$ est l'inclinaison de la conduite, $\lambda$ est le coefficient de perte de charge linéaire, $t$ est le temps et $x$ est l'abscisse le long de l'axe de la conduite.

Les deux variables principales sont la vitesse de l'écoulement $V$ et la pression du fluide $p$. La masse volumique du fluide $\rho$ et la section de la conduite $A$ peuvent s'exprimer en fonction de la pression du fluide.

\subsection{Loi de comportement élastique non-linéaire}

Supposons que le comportement des parois de la conduite soit élastique, isotrope et obéisse à la loi classique de Hooke, les déformations radiales sont petites $\left(0,9<R / R_{0}<1,1\right)$ et le coefficient de Poisson $\nu$ est proche de 0,5 . Le volume de la paroi de la conduite par unité de longueur reste alors constant et on peut écrire :

$$
e R=e_{0} R_{0}
$$


où $e$ et $R$ sont respectivement l'épaisseur et le rayon de la conduite à la pression $p$. L'indice 0 fait référence aux conditions initiales.

Afin d'établir une relation permettant d'exprimer la pression du fluide en fonction de la section de la conduite $\left(A=\pi R^{2}\right)$, nous nous basons sur la loi de comportement élastique non-linéaire, pour laquelle le module de Young obéit à la relation proposée par Wetterer et Kenner [3] :

$$
E / E_{0}=\left(R / R_{0}\right)^{\delta}
$$

où $R$ est une longueur caractéristique du matériau et $\delta$ est un paramètre de rigidité de la conduite défini par :

$-\delta=0$ pour les matériaux à comportement élastique linéaire;

$-\delta \neq 0$ pour les matériaux à comportement élastique non-linéaire.

En négligeant l'inertie radiale de la paroi de la conduite, la contrainte circonférentielle sera exprimée par :

$$
\sigma_{\phi}=\frac{p R}{e}
$$

Les lois de Hooke combinées avec la relation (4), permettent d'écrire :

$$
\mathrm{d} \sigma_{\phi}=E \mathrm{~d} \varepsilon_{\phi} \Leftrightarrow \quad \mathrm{d}\left(\frac{p R}{e}\right)=E \frac{\mathrm{d} R}{R}=E_{0}\left(\frac{R}{R_{0}}\right)^{\delta} \frac{\mathrm{d} R}{R}
$$

En tenant compte de (3), l'intégration de l'équation (6) donne :

$$
p=\frac{A_{0}}{A}\left[\frac{\sqrt{\pi} E_{0} e_{0}}{\delta \sqrt{A_{0}}}\left(\left(\frac{A}{A_{0}}\right)^{\delta / 2}-1\right)+p_{0}\right] \text { avec } \delta \neq 0
$$

\subsection{Modèle de cavitation à bulles de vapeur}

Dans les circuits hydrauliques, lorsque dans certaines zones, la pression devient inférieure à la pression de saturation, il se produit brusquement des cavités remplies de vapeur. Ce phénomène est connu sous le nom de cavitation de vapeur.

Pour étudier le phénomène de cavitation nous partons de l'idée générale de formation des bulles de vapeur dès que la pression devient inférieure à une certaine pression $p_{\mathrm{v}}$. Il s'agit de la loi de Henry dont la forme doit être modifiée.

En considérant le fluide comme étant un mélange homogène de liquide-vapeur, la masse volumique de ce mélange sera exprimée en fonction de la masse volumique du liquide, $\rho_{\mathrm{l}}$, et celle du vapeur, $\rho_{\mathrm{v}}$, par :

$$
\rho=\alpha \rho_{\mathrm{l}}+(1-\alpha) \rho_{\mathrm{V}}
$$

où $\alpha=\frac{\vartheta_{1}}{\vartheta_{\mathrm{m}}}$ est la fraction volumique du liquide dans le mélange. $\vartheta_{\mathrm{l}}$ et $\vartheta_{\mathrm{m}}$ représentent les volumes du liquide et du mélange respectivement.

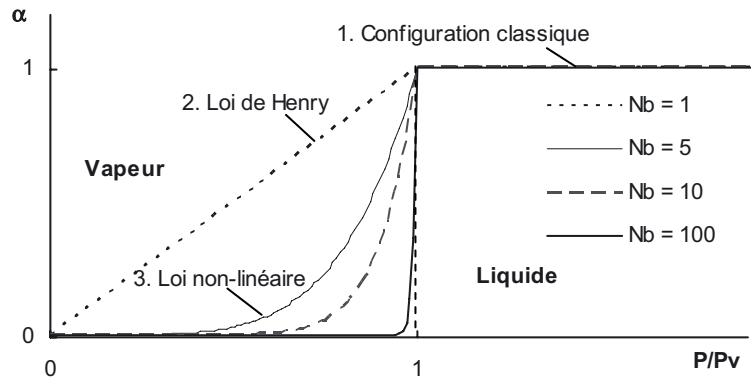

Fig. 1. Modèle de cavitation à bulles de vapeur.

La configuration classique de la cavitation de vapeur suggère de faire représenter la fraction volumique du liquide par la fonction :

$$
\alpha=\left\{\begin{array}{lll}
1 & \text { si } & p>p_{\mathrm{v}} \\
0 & \text { si } & p \leq p_{\mathrm{v}}
\end{array}\right.
$$

Cette fonction, reproduite sur la figure 1 par la courbe 1 , est discontinue à $p=p_{\mathrm{v}}$. Il en résulte des instabilités lors du calcul numérique.

Le modèle proposé suppose que la pression tombe légèrement en dessous de la pression de vapeur. Toutefois, la chute négative de la pression prend seulement quelques millisecondes puis elle remonte de nouveau à la pression de vapeur. Par analogie avec la loi de Henry pour le dégazage de l'air dissous [1] (courbe 2 de Fig. 1), la fraction volumique du liquide sera définie par la formule approchée suivante :

$$
\alpha=\left\{\begin{array}{lll}
1 & \text { si } & p>p_{\mathrm{v}} \\
\left(\frac{p}{p_{\mathrm{v}}}\right)^{N b} & \text { si } & p \leq p_{\mathrm{v}}
\end{array}\right.
$$

où $N b$ est un nombre assez grand pour qu'on puisse se rapprocher de la configuration classique (courbe 3 de Fig. 1).

Lorsque la pression est supérieure à la pression de vapeur, le fluide est purement liquide et on a $\alpha=1$. La masse volumique du mélange est calculée à partir de la relation empirique suivante [4] :

$$
\rho=\rho_{\mathrm{l}}=\rho_{\mathrm{l}_{0}}\left(\frac{p+b}{a}\right)^{1 / n} \quad \text { avec } \quad p>p_{\mathrm{v}}
$$

L'indice 0 fait référence aux conditions de l'écoulement permanent initial à $p_{0}=1$ bar. Pour l'eau $n=7, a=$ 3001 bar, $b=3000$ bar, $\rho_{\mathrm{l}_{0}}=1000 \mathrm{~kg} . \mathrm{m}^{-3}$

Dans la zone de cavitation, où $p \leq p_{\mathrm{v}}$, on aura :

$$
\rho=\left(\frac{p}{p_{\mathrm{v}}}\right)^{N b} \rho_{\mathrm{l}}+\left(1-\left(\frac{p}{p_{\mathrm{v}}}\right)^{N b}\right) \rho_{\mathrm{v}}
$$

Afin d'avoir un mélange homogène et de pouvoir appliquer la relation (8), les bulles de vapeur sont supposées uniformément distribuées dans le liquide et elles ont un comportement isothermique. Dans ces conditions, 
la relation entre la pression et la masse volumique de la vapeur s'exprime à partir de la loi d'état pour la vapeur qui s'écrit :

$$
p / \rho_{\mathrm{v}}=\Gamma_{\mathrm{v}} T=\text { const. }
$$

où $\Gamma_{\mathrm{v}}$ est la constante de vapeur considérée comme un gaz parfait $\left(\Gamma_{\mathrm{v}}=491 \mathrm{~J} \cdot \mathrm{kg}^{-1} \cdot \mathrm{K}^{-1}\right)$ et $T$ est la température absolue.

En utilisant les équations (11) et (13), l'expression (12) de la masse volumique s'écrit :

$$
\rho=\left(\frac{p}{p_{\mathrm{v}}}\right)^{N b} \rho_{\mathrm{l}_{0}}\left(\frac{p+b}{a}\right)^{1 / n}+\left(1-\left(\frac{p}{p_{\mathrm{v}}}\right)^{N b}\right) \frac{p}{\Gamma_{\mathrm{v}} T},
$$$$
\text { avec } p \leq p_{\mathrm{v}}
$$

\subsection{Formulation conservative}

Les équations (1) et (2) sont réécrites sous une forme conservative comme suit :

$$
\begin{gathered}
\frac{\partial U}{\partial t}+\frac{\partial W}{\partial x}=0 \\
\frac{\partial W}{\partial t}+\frac{\partial\left(B+W^{2} / U\right)}{\partial x}=G
\end{gathered}
$$

Les variables conservatives principales du système d'équations (15) et (16) sont $U=\rho A$ et $W=\rho A V=U V$, et les fonctions $B$ et $G$ sont définies par $B=\int A \mathrm{~d} p$ et $G=U g \sin \gamma-\lambda \sqrt{\pi} W|W| /(4 U \sqrt{A})$.

Pour les conduites déformables à loi de comportement non-linéaire :

$$
\begin{array}{r}
B=\frac{E_{0} e_{0} \sqrt{\pi A_{0}}}{\delta}\left[\left(1-\frac{2}{\delta}\right)\left(\frac{A}{A_{0}}\right)^{\delta / 2}+\left(1-\frac{p_{0} \delta \sqrt{A_{0}}}{E_{0} e_{0} \sqrt{\pi}}\right)\right. \\
\left.\times \ln \left(\frac{A}{A_{0}}\right)\right] \text { avec } \delta \neq 0 \quad(17
\end{array}
$$

\section{Résolution numérique}

\subsection{Schéma de Lax-Wendroff}

Suivant la technique décrite par Peret et Taylor [5], le système d'équations aux dérivées partielles (15) et (16) est résolu par le schéma numérique à deux pas de LaxWendroff :

- Premier pas (ou phase de prédiction) :

$$
\begin{aligned}
U_{i+1 / 2}^{k+1 / 2}= & \frac{1}{2}\left[\left.U\right|_{i} ^{k}+\left.U\right|_{i+1} ^{k}\right]-\frac{\Delta t}{2 \Delta x}\left[\left.W\right|_{i+1} ^{k}-\left.W\right|_{i} ^{k}\right] \\
\left.W\right|_{i+1 / 2} ^{k+1 / 2}= & \frac{1}{2}\left[\left.W\right|_{i} ^{k}+\left.W\right|_{i+1} ^{k}\right]-\frac{\Delta t}{2 \Delta x}\left[\left.\left(B+W^{2} / U\right)\right|_{i+1} ^{k}\right. \\
& \left.-\left.\left(B+W^{2} / U\right)\right|_{i} ^{k}\right]+\frac{\Delta t}{4}\left(\left.G\right|_{i+1} ^{k}+\left.G\right|_{i} ^{k}\right)
\end{aligned}
$$

- Deuxième pas (ou phase de correction) :

$$
\begin{aligned}
\left.U\right|_{i} ^{k+1}= & \left.U\right|_{i} ^{k}-\frac{\Delta t}{\Delta x}\left[\left.W\right|_{i+1 / 2} ^{k+1 / 2}-\left.W\right|_{i-1 / 2} ^{k+1 / 2}\right] \\
\left.W\right|_{i} ^{k+1}= & \left.W\right|_{i} ^{k}-\frac{\Delta t}{\Delta x}\left[\left.\left(B+W^{2} / U\right)\right|_{i+1 / 2} ^{k+1 / 2}\right. \\
& \left.-\left.\left(B+W^{2} / U\right)\right|_{i-1 / 2} ^{k+1 / 2}\right]+\left.\Delta t G\right|_{i} ^{k}
\end{aligned}
$$

où $i$ et $k$ sont des indices pour le pas d'espace et le pas de temps, $\Delta x=L / N$ est le pas d'espace, et $\Delta t$ est le pas de temps conditionné par le critère de stabilité de Courant Friederich Levy [4] :

$$
\frac{\Delta t}{\Delta x} \leqslant \frac{1}{(|V|+C)_{\max }}
$$

où $C$ est la célérité des ondes de pression dont l'expression est développée dans l'annexe A.

\subsection{Calcul de la pression et de la vitesse}

Si la valeur de la vitesse $V$ peut être directement déduite du quotient $W / U$, il n'en est pas de même pour la valeur de $p$ qui sera calculée en résolvant par la méthode de Newton, l'équation algébrique $\rho(p) A(p)-U=0$. Ceci conduit à l'algorithme itératif suivant [6] :

$$
\left\{\begin{array}{l}
A_{0}=\left.A\right|_{i} ^{k}, \quad p_{0}=\left.p\right|_{i} ^{k} \\
A_{\mathrm{m}+1}=A_{\mathrm{m}}-\rho_{\mathrm{m}} A_{\mathrm{m}} C_{\mathrm{m}}^{2}\left[1-\frac{\left.\rho A\right|_{i} ^{k+1}}{\rho_{\mathrm{m}} A_{\mathrm{m}}}\right] \frac{2 \sqrt{A_{0}}}{\sqrt{\pi} E_{0} e_{0} F_{\mathrm{m}}} \\
p_{\mathrm{m}+1}=p\left(A_{\mathrm{m}+1}\right) \\
\rho_{\mathrm{m}+1}=\rho\left(p_{\mathrm{m}+1}\right)
\end{array}\right.
$$

$\rho, A, C$ et $F$ sont définies par les expressions (11), (14), (7), (A.3) et (A.4) respectivement.

\subsection{Conditions initiales}

Les conditions initiales sont données par le régime permanent initial obtenu en résolvant le système d'équations différentielles (15) et (16) en annulant les termes en $\partial / \partial t$. On obtient le système d'équations différentielles ordinaires suivant :

$$
\left\{\begin{array}{c}
\frac{\mathrm{d} W}{\mathrm{~d} x}=\frac{\mathrm{d}(\rho A V)}{\mathrm{d} x}=0 \Rightarrow \rho A V=W_{0}=\text { const. } \\
W_{0}^{2} \frac{\mathrm{d}}{\mathrm{d} x}\left(\frac{1}{\rho A(p)}\right)+A(p) \frac{\mathrm{d} p}{\mathrm{~d} x}+\frac{\lambda \sqrt{\pi} W_{0}^{2}}{4 \rho A(p) \sqrt{A(p)}} \\
-\rho A(p) g \sin \gamma=0
\end{array}\right.
$$

La deuxième équation de ce système est résolue par la méthode de Runge-Kutta. 


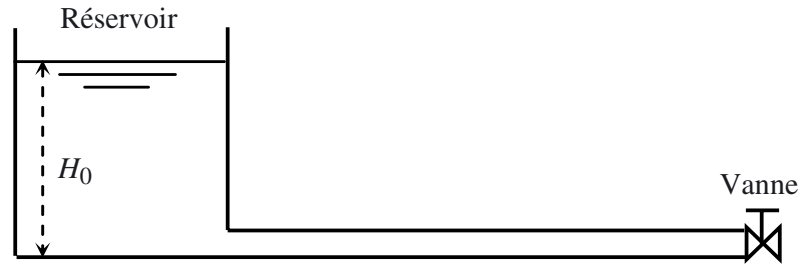

Fig. 2. Schéma de l'installation hydraulique.

Tableau 1. Caractéristiques de l'installation.

\begin{tabular}{lc}
\hline Longueur de la conduite & $L=1 \mathrm{~m}$ \\
Diamètre de la conduite & $D=18 \mathrm{~mm}$ \\
Épaisseur de la conduite & $e=1 \mathrm{~mm}$ \\
Hauteur de pression & $H_{0}=1,07 \mathrm{~m}$ \\
Vitesse initiale & $V_{0}=0,8 \mathrm{~m} \cdot \mathrm{s}^{-1}$ \\
Coefficient de charge linéaire & $\lambda=0,02$ \\
Coefficient de Poisson & $\nu=0,5$ \\
Module de Young & $E_{0}=2,22 \mathrm{MPa}$ \\
Masse volumique du fluide & $\rho=1000 \mathrm{~kg} \cdot \mathrm{m}^{-3}$ \\
\hline
\end{tabular}

\subsection{Conditions aux limites}

Pour les écoulements transitoires en conduites, les conditions aux limites sont des lois imposées à la pression et à la vitesse de l'écoulement aux deux extrémités de la conduite.

Dans cette étude, l'écoulement transitoire est provoqué par une fermeture d'une vanne à l'extrémité aval de la conduite $(x=L)$. À cette section, si la fermeture est instantanée alors $\left.V\right|_{N+1} ^{k+1}=V(L, t)=0$. Sinon, la vitesse est exprimée par :

$$
\left.V\right|_{N+1} ^{k+1}=V_{0} \tau_{\text {vanne }} \sqrt{\frac{\left.H\right|_{N+1} ^{k+1}}{H_{\text {vanne }}}}
$$

où $\tau_{\text {vanne }}$ est l'ouverture adimensionnelle de la vanne exprimée en terme du temps de fermeture $t_{c}$ par :

$$
\tau_{\text {vanne }}= \begin{cases}1-t / t_{\mathrm{c}} & \text { si } \quad t \leq t_{\mathrm{c}} \\ 0 & \text { si non }\end{cases}
$$

À l'extrémité amont de la conduite $(x=0$ et $t>0)$ la condition est donnée par un réservoir à pression constante $\left.p\right|_{1} ^{k+1}=p(0, t)=p_{0}(0)$ ou $\left.H\right|_{1} ^{k+1}=H(0, t)=H_{0}(0)$.

\section{Applications et résultats}

\subsection{Coup de bélier sans cavitation}

Considérons l'exemple d'une installation hydraulique (Fig. 2) constituée d'une conduite horizontale liée en amont à un réservoir à niveau constant et en aval à une vanne. Les caractéristiques de cette installation sont indiquées dans le tableau 1. Le régime transitoire est provoqué par la fermeture brusque de la vanne.

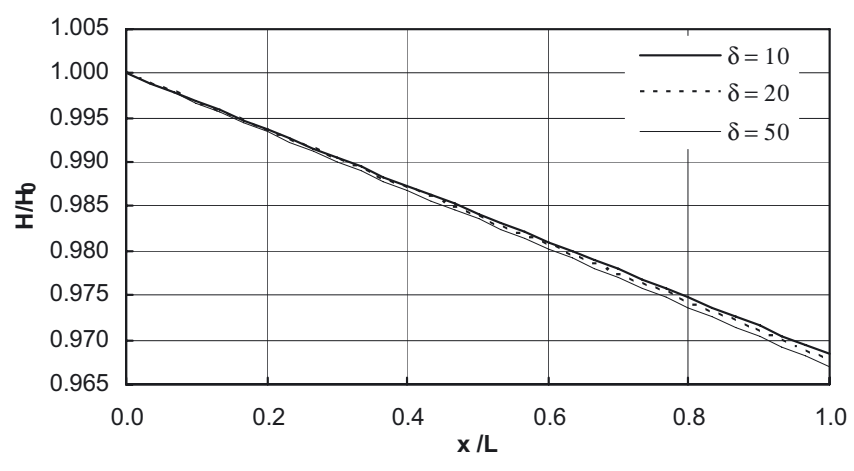

Fig. 3. Hauteur de pression en régime permanent en fonction de $\delta$.

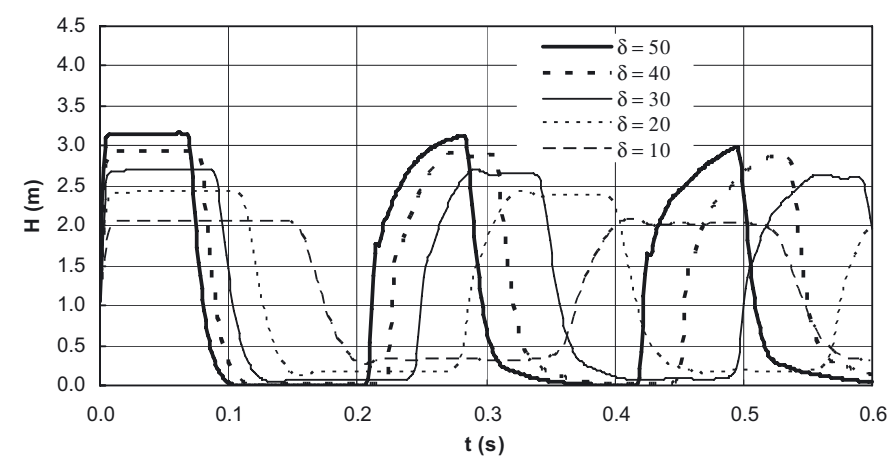

Fig. 4. Hauteur de pression à la vanne en régime transitoire en fonction de $\delta$ (résultats issus du modèle numérique proposé).

\section{- Influence du paramètre $\delta$}

Les courbes de la hauteur de pression en régime permanent, pour différentes valeurs de $\delta$, sont représentées sur la figure 3 . Une légère augmentation des pertes de charge est observée lorsque $\delta$ augmente.

La figure 4 représente l'évolution de la hauteur de pression à la vanne en régime transitoire pour différentes valeurs de $\delta$. Nous remarquons que les courbes de la hauteur de pression subissent des oscillations avant de s'amortir progressivement à cause des pertes de charge. Nous constatons aussi que les amplitudes de cette hauteur augmentent quand $\delta$ augmente. En effet, plus $\delta$ est [plus] grand, plus le matériau se rigidifie, d'où des pics de pression supérieurs.

\section{- Influence du module d'élasticité de Young}

La figure 5 représente l'évolution de la hauteur de pression à la vanne pour différentes valeurs du module de Young. Le paramètre de rigidité $\delta$ est égal à 10 .

Nous remarquons que les amplitudes de la hauteur de pression augmentent quand $E$ croît. Ceci peut être interprété par le fait que les pics de pression sont d'autant plus grands que le matériau est rigide. 


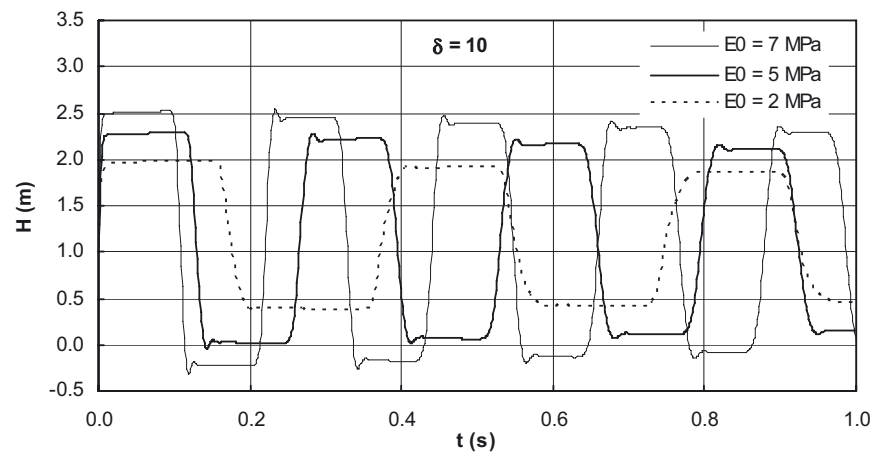

Fig. 5. Hauteur de pression à la vanne en fonction du module de Young (résultats issus du modèle numérique proposé).

Tableau 2. Caractéristiques des conduites.

\begin{tabular}{cccc}
\hline Matériaux & $\begin{array}{c}\text { Diamètre } \\
\text { intérieur }(\mathrm{mm})\end{array}$ & $\begin{array}{c}\text { Épaisseur } \\
(\mathrm{mm})\end{array}$ & $\begin{array}{c}\text { Célérité } \\
\left(\mathrm{m} \cdot \mathrm{s}^{-1}\right)\end{array}$ \\
\hline PEMD PN6 & 44,2 & 2,9 & 320 \\
PEMD PN10 & 40,8 & 4,6 & $400-410$ \\
PVC-U PN10 & 45,2 & 2,4 & $400-410$ \\
\hline
\end{tabular}

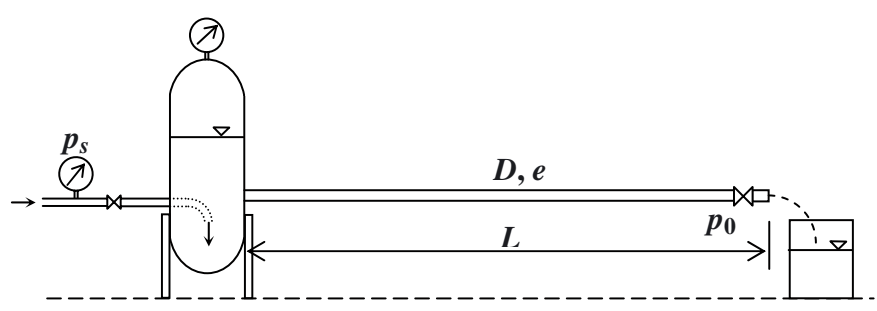

Fig. 6. Schéma de l'installation expérimentale.

\subsection{Coup de bélier avec cavitation, validation avec l'expérience de Mitosek}

Le travail expérimental de Mitosek [7] est utilisé pour évaluer et valider le modèle de cavitation à bulles de vapeur dans le cas des écoulements transitoires avec cavitation de vapeur dans les conduites en matériaux plastiques.

L'expérience de Mitosek consiste à un écoulement dans une conduite circulaire de longueur $L=15 \mathrm{~m}$. Le tableau 2 indique les caractéristiques des conduites utilisées ainsi que la célérité mesurée des ondes de pression $\left(C=4 L / T_{\mathrm{m}}\right) \cdot T_{\mathrm{m}}$ est la période de l'onde de pression mesurée sur la courbe expérimentale.

Les tests ont été réalisés en utilisant l'appareil expérimental représenté sur la figure 6 . Les conduites à tester sont alimentées par un réservoir lié au réseau de distribution d'eau. La pression $p_{\mathrm{s}}$ de l'eau à l'entrée de l'installation est constante $\left(p_{\mathrm{s}}=0,65 \mathrm{MPa}\right)$. La variation de $V_{0}$ de 0,5 à $2 \mathrm{~m} . \mathrm{s}^{-1}$ permet d'avoir différentes pressions $p_{0}$ et différentes surpressions $\Delta p$ pour la première onde de pression à obtenir.

Durant ces tests la température est maintenue constante à $8{ }^{\circ} \mathrm{C}$. Ceci est important vu l'influence de la température sur les propriétés mécaniques des conduites plastiques. La pression de vapeur, $p_{\mathrm{v}}$, est égale à $1003 \mathrm{~Pa}$.

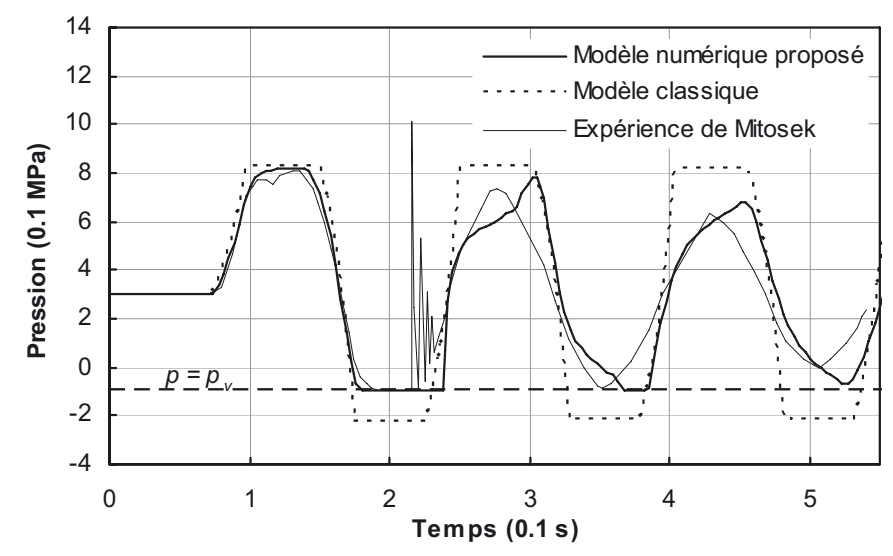

Fig. 7. Évolution de la pression à la vanne (conduite en PVC$\left.\mathrm{U}, V_{0}=1,35 \mathrm{~m} \cdot \mathrm{s}^{-1}, p_{0}=0,299 \mathrm{MPa}, \delta=-50\right)$.

L'écoulement transitoire est provoqué par la fermeture brusque d'une vanne montée à l'extrémité aval de la conduite. Le temps de fermeture $t_{\mathrm{c}}$ est de l'ordre de 0,02 à $0,025 \mathrm{~s}$ dans tous les tests.

\section{- Choix du paramètre de rigidité $\delta$}

Le choix du paramètre $\delta$ dépend de la loi du comportement élastique non-linéaire du matériau de la conduite. En supposant le coefficient de Poisson constant, l'étude se ramène à la détermination de la seule loi du module de Young, équation (4), en examinant son comportement en traction simple.

Une série de mesures faite par Hadj-Tä̈eb [8] sur des éprouvettes en différentes matériaux élastiques (caoutchouc, polyéthylène, PVC) a montré que les valeurs du paramètre $\delta$ pour ces matériaux sont négatives $(-100$ pour le caoutchouc, -40 pour le polyéthylène et 0 pour le PVC).

Pour l'exemple de l'expérience de Mitosek, une valeur de $\delta$ égale à -50 permet d'obtenir des résultats de calcul qui correspondent mieux à ceux de l'expérience.

\section{- Comparaison des résultats}

Les figures 7, 8 et 9 représentent une comparaison de l'évolution de la pression à la vanne obtenue par le modèle numérique proposé avec celle obtenue par l'expérience de Mitosek pour différents types de matériaux de conduites. Le calcul numérique est effectué avec une valeur du nombre $N b$ égale à 100 .

Dans tous les cas envisagés, la pression tombe à la pression de vapeur au moins pour la première onde négative du coup de bélier produisant ainsi le phénomène de cavitation de vapeur.

La figure 7 montre la grande différence entre les résultats du modèle classique du coup de bélier (sans modélisation de la cavitation) et ceux de l'expérience. La pression minimale est bien en dessous de la pression de vapeur du liquide. L'hypothèse d'un fluide homogène et continu faite dans le modèle classique n'est plus respectée quand la pression tombe en dessous de la pression de vapeur. C'est pourquoi le modèle classique, sans test sur la 


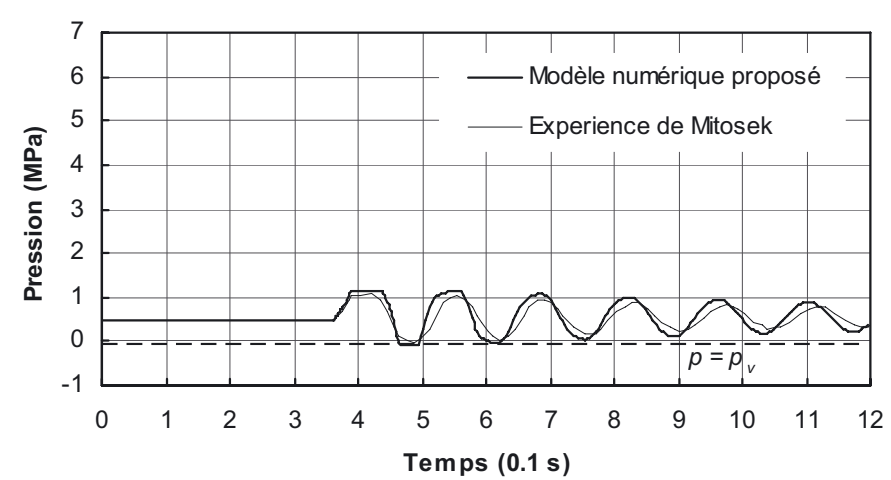

Fig. 8. Évolution de la pression à la vanne (conduite en PEMD PN10, $\left.V_{0}=1,7 \mathrm{~m} \cdot \mathrm{s}^{-1}, p_{0}=0,5 \mathrm{MPa}, \delta=-50\right)$.

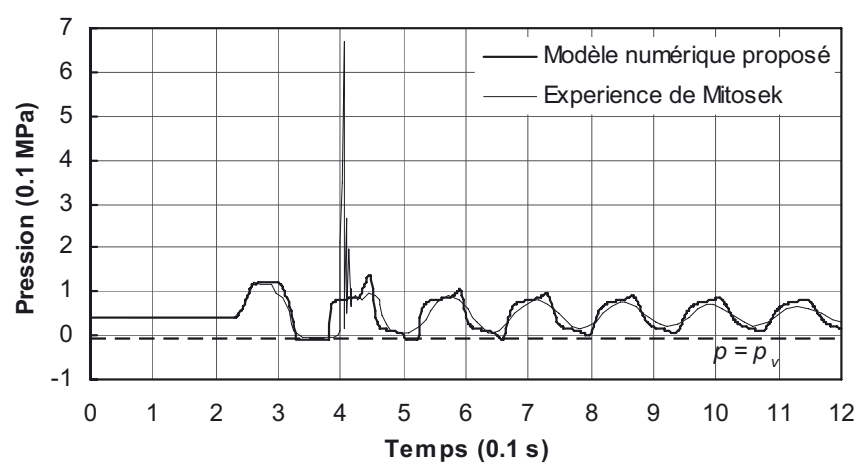

Fig. 9. Évolution de la pression à la vanne (conduite en $\mathrm{PE}-$ MD PN10, $\left.V_{0}=2 \mathrm{~m} \cdot \mathrm{s}^{-1}, p_{0}=0,4 \mathrm{MPa}, \delta=-50\right)$.

pression, est incapable de décrire le phénomène de cavitation de vapeur. Par contre, nous remarquons l'accord raisonnable entre les résultats issus du modèle de cavitation à bulles de vapeur proposé et ceux de l'expérience.

Dans ses expériences, Mitosek a pu enregistrer des pics de pression suite à la fermeture de la zone de cavitation. Ces pics de pression sont plus importants que les surpressions dues à la première onde positive du coup de bélier (pression de Joukovski). Les résultats du modèle proposé ne présentent pas de tels pics de pression. Bien que l'implosion des structures cavitantes est connue pour générer des surpressions parfois très élevées, ces pics semble être irréalistes et peuvent être dus à la sensibilité des capteurs utilisés pour mesurer les pressions.

\section{- Influence du paramètre de rigidité $\delta$}

Les figures 10 et 11 représentent respectivement l'évolution de la pression à la vanne et au milieu de la conduite pour différentes valeurs du paramètre de rigidité $\delta$. Quand $\delta$ diminue, nous remarquons une légère diminution de l'amplitude de pression, une diminution de la célérité des ondes de pression (Fig. 12), ainsi que le retard de l'apparition du phénomène de cavitation de vapeur. Ceci peut être expliqué par l'augmentation de l'élasticité des parois de la conduite quand $\delta$ diminue.

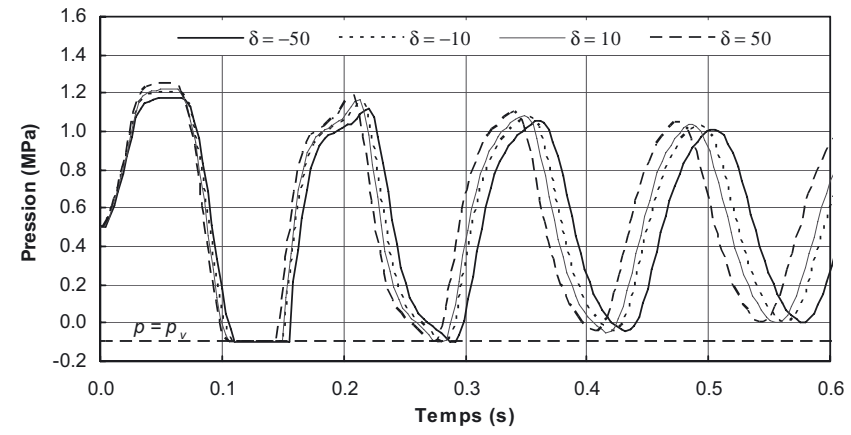

Fig. 10. Évolution de la pression à la vanne, résultats issus du modèle numérique proposé, (conduite en PE-MD PN10, $\left.V_{0}=1,7 \mathrm{~m} \cdot \mathrm{s}^{-1}, p_{0}=0,5 \mathrm{MPa}\right)$.

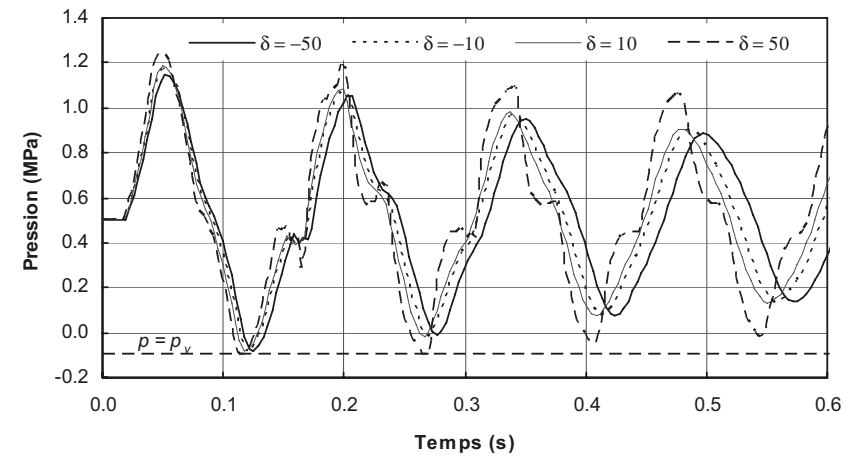

Fig. 11. Évolution de la pression au milieu de la conduite, résultats issus du modèle numérique proposé, (conduite en PEMD PN10, $\left.V_{0}=1,7 \mathrm{~m} \cdot \mathrm{s}^{-1}, p_{0}=0,5 \mathrm{MPa}\right)$.

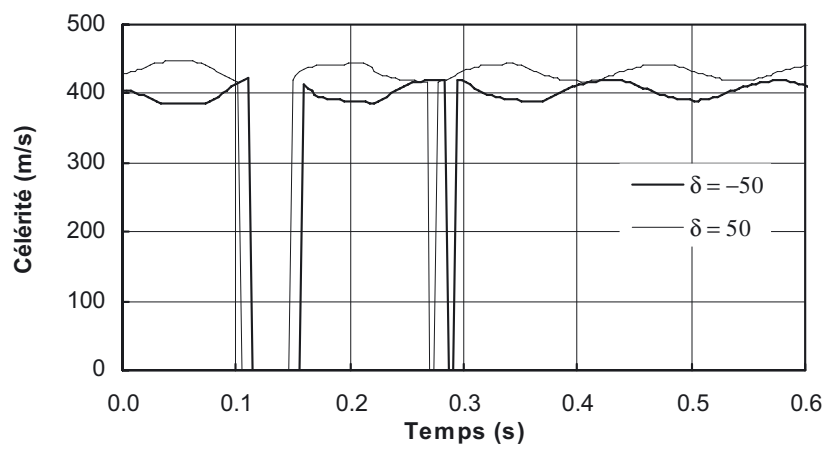

Fig. 12. Évolution de la célérité en régime transitoire, résultats issus du modèle numérique proposé.

\section{- Influence du nombre $\mathrm{Nb}$}

Les courbes de la pression à la vanne obtenues par le modèle proposé pour différentes valeurs du nombre $\mathrm{Nb}$ sont représentées sur la figure 13. Des instabilités de calcul numérique persistent lorsque la valeur de $N b$ est petite $(N b=1)$. Dans ce cas, la modélisation du phénomène de cavitation n'est pas introduite dans la simulation avec suffisamment de précision $(\alpha \approx 0,97)$. Pour des valeurs de $N b$ plus grandes les instabilités disparaissent et les résultats sont nettement meilleurs. En effet, la valeur atteinte de $\alpha$ est égal à 0,13 pour $N b=10$ et 0,047 pour $N b=100$. 


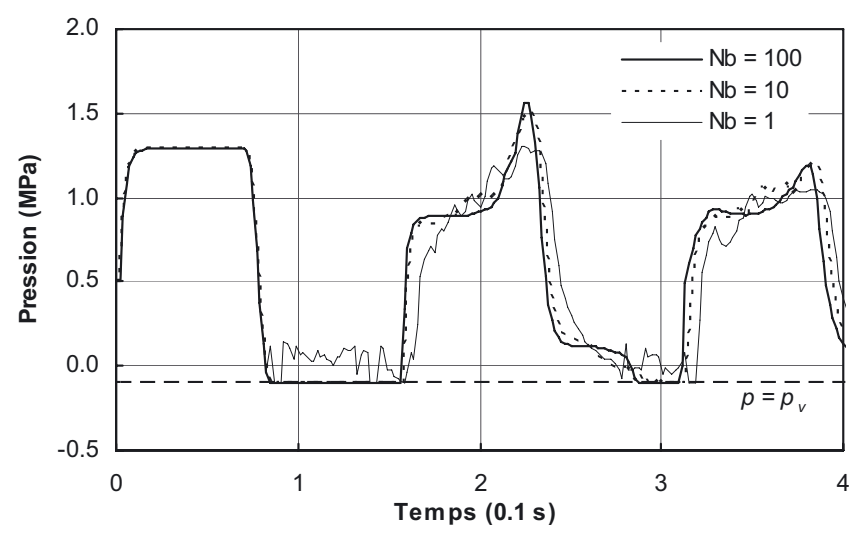

Fig. 13. Influence du nombre $N b$ sur les résultats de simulation, (conduite en PE-MD PN10, $V_{0}=2 \mathrm{~m} . \mathrm{s}^{-1}, p_{0}=0,4 \mathrm{MPa}$, $\delta=-50)$.

\section{Conclusion}

Le modèle numérique présenté nous a permis d'étudier l'interaction fluide-structure dans les écoulements transitoires en conduites élastiques avec cavitation de vapeur.

L'avantage de ce modèle est qu'il donne une description raisonnable de l'allure générale du phénomène étudié et reproduit avec satisfaction l'évolution des ondes de pression en comparaison avec l'expérience.

La présente étude montre l'influence du comportement de la paroi de la conduite dans les phénomènes de coup de bélier et de cavitation de vapeur. Nous avons pu constater que l'évolution de ces phénomènes est étroitement liée au module d'élasticité de Young et au paramètre de rigidité utilisé dans la loi de comportement non-linéaire.

Sur le plan calcul numérique, l'étude montre bien l'efficacité de la méthode de Lax-Wendroff pour simuler convenablement les phénomènes étudiés.

\section{Annexe A : Expression de la célérité}

D'après Stuckenbruck et al. [9], on peut exprimer la célérité des ondes de pression dans les écoulements en conduites par la formule :

$$
C=\left(\frac{\partial \rho}{\partial p}+\frac{\rho}{A} \frac{\partial A}{\partial p}\right)^{-1 / 2}
$$

En tenant compte des relations (7), (11) et (14), on obtient :

$$
\frac{1}{C^{2}}=\frac{1}{C_{0}^{2}}+\frac{\rho D_{0}}{E_{0} e_{0} F}
$$

où $C_{0}$ est la célérité des ondes sonores dans le fluide :

$$
\begin{aligned}
C_{0}= & \left(\frac{\partial \rho}{\partial p}\right)^{-1 / 2} \\
& =\left\{\begin{array}{l}
\sqrt{\frac{n(p+b)}{\rho_{\mathrm{l}}}} \text { si } p>p_{\mathrm{v}} \\
\frac{1}{\rho}\left[\frac{\Gamma_{\mathrm{v}} T}{p^{2}}\left(1-\left(\frac{p}{p_{\mathrm{v}}}\right)^{N b}\right)+\frac{\Gamma_{\mathrm{v}} T N b}{p_{\mathrm{v}}^{2}}\left(\frac{p}{p_{\mathrm{v}}}\right)^{N b-2}\right. \\
\left.-\frac{N b}{\rho_{\mathrm{l}} p_{\mathrm{v}}}\left(\frac{p}{p_{\mathrm{v}}}\right)^{N b-1}+\left(\frac{p}{p_{\mathrm{v}}}\right)^{N b} / \rho_{\mathrm{l}} n(p+b)\right]^{-1 / 2}
\end{array}\right.
\end{aligned}
$$

et $F$ est une fonction représentant le comportement élastique non-linéaire des parois de la conduite :

$$
F=\left(\frac{A}{A_{0}}\right)^{(\delta / 2)-1}-\frac{2 \sqrt{A_{0}}}{E_{0} e_{0} \sqrt{\pi}} p
$$

\section{Références}

[1] E. Hadj-Taïeb, T. Lili, Les écoulements transitoires dans les conduites déformables avec dégazage de l'air dissous, La Houille Blanche (2001) 99-107

[2] V.L. Streeter, E.B. Wylie, Hydraulic transient, F.E.B. press, Ann Arbor, 1983

[3] E. Wetterer, Th. Kenner, Studie ueber die verschiedenen definitionen des elastizitaets modulus in der haemodynamik, Zeit. Biol. 114 (1963) Munich

[4] R. Courant, K.O. Friedrichs, Supersonic flow and shock waves, Interscience publishers Inc., New York, 1948

[5] A. Lerat, R. Peyret, Sur le choix des schémas aux différences du second ordre fournissant des profils de choc sans oscillations, C. R. Acad. Sci. Paris 277 (1973) 363-366

[6] E. Hadj-Taïeb, T. Lili, Transient flow of homogeneous gasliquid mixtures in pipeline, Int. J. Numerical Methods for Heat and Fluid Flow 8 (1998) 350-368

[7] M. Mitosek, Study of cavitation due to water hammer in plastics pipes, Plastics, rubber and composites processing and applications 26 (1997) 324-329

[8] E. Hadj-Taïeb, Écoulements transitoires dans les conduites déformables avec cavitation de vapeur et dégazage de l'air dissous, Thèse de doctorat d'état en sciences-physiques, Laboratoire de mécanique des fluides, Faculté des Sciences de Tunis, 1999

[9] S. Stuckenbruck, D.C. Wiggert, R.S. Otwell, The influence of pipe motion on acoustic wave propagation, Transactions of the ASME 107 (1985) 518-522 